\title{
Quantum partition noise of photo-created electron-hole pairs
}

\author{
L.-H. Reydellet, f P. Roche, and D. C. Glattlif \\ Service de Physique de l'Etat Condensé, \\ CEA Saclay, F-91191 Gif-Sur-Yvette, France \\ B. Etienne and Y. Jin \\ Laboratoire de Photonique et de Nanostructures, \\ CNRS, Route de Nozay, F-91460 Marcoussis, France
}

(Dated: November 12, 2018)

\begin{abstract}
We show experimentally that even when no bias voltage is applied to a quantum conductor, the electronic quantum partition noise can be investigated using $\mathrm{GHz}$ radiofrequency irradiation of a reservoir. Using a Quantum Point Contact configuration as the ballistic conductor we are able to make an accurate determination of the partition noise Fano factor resulting from the photo-assisted shot noise. Applying both voltage bias and rf irradiation we are able to make a definitive quantitative test of the scattering theory of photo-assisted shot noise.

PACS numbers: 73.23.-b, 73.23.Ad, 73.50.Pz, 73.50.Td
\end{abstract}

Can electron quantum partition noise be observed without net electron transport? In this letter we investigate the shot noise of a ballistic conductor under radiofrequency irradiation. We show experimentally that photo-created electron hole pairs do generate shot noise even when no net current flows through the conductor.

Current noise measurements associated with a d.c. current have revealed many important phenomena in mesoscopic physics on the last decade. Fundamental current fluctuations in a quantum conductor out of equilibrium, called shot noise, are a sensitive probe of both the charge and the statistics of the carriers [1, 2]. This approach has led to the observation of the quantum suppression of shot noise in ballistic conductors due to the Fermi statistics 3, 4, the Laughlin quasiparticle charge in the fractional quantum Hall regime [5, 6], the doubling of shot noise resulting from Andreev reflections at Normal-Superconductor interfaces $[7,8]$ and the enhanced noise due to multiple Andreev reflections [9, 10, the reduction factor associated with the distribution of transmission probabilities in diffusive or chaotic quantum conductors 11, 12, 13, 14].

In general shot noise is produced when the quantum conductor is driven out of equilibrium. In all the work quoted above, non equilibrium was obtained by applying an electrochemical potential difference between the electron reservoirs (or contacts) resulting in a net current. Here, the origin is simple to understand. Consider for simplicity a single mode quantum conductor at low temperature with say the left reservoir biased by an electrochemical potential difference $\mathrm{eV}$ with respect to the right. The left reservoir emits regularly electrons toward the conductor at a frequency $e V / h$ as a result of the Fermi statistics giving a incoming current $I_{0}=e(e V / h)$. If $D$ is the electron transmission probability, the transmitted current $I=D I_{0}$ gives the Landauer conductance $G=D e^{2} / h$. As the regular injection of electrons is noiseless, the only source of shot noise corresponds to the quantum partition noise generated by electrons either transmitted or reflected. The resulting current fluctuations for a frequency bandwidth $\Delta f$ are $\overline{\Delta I^{2}}=2 e I_{0} D(1-D) \Delta f$, where the term $D(1-D)$ is the variance of the binomial statistics of the partitioning 15.

Non equilibrium shot noise can however be produced when no voltage bias and hence no mean current flows through the conductor. For example, heating one reservoir is expected to generate thermal noise but also shot noise with a $D(1-D)$ dependence reflecting partitioning even when no thermo-electric current is generated [2, 16. Another non equilibrium situation occurs when photons irradiate one side of the quantum conductor (say the left one). This is the regime addressed in this letter. To understand the mechanism, consider an electron emitted from the left reservoir with an energy $\epsilon \leq h \nu$ below the Fermi energy. The electron can be either pumped to an energy $h \nu-\varepsilon$ above the Fermi energy with probability $\mathcal{P}_{1}$ or unpumped with probability $\mathcal{P}_{0}$. Unpumped electrons cannot generate current fluctuations as the right reservoir also emits electrons at the same energy. The Pauli principle imposes that both right and left outgoing states be filled with one electron leading to no current and hence no fluctuation. However the photo-pumped incoming electrons and holes do generate noise: the right reservoir does not emit electrons nor holes at energies $h \nu-\epsilon$ and $-\epsilon$ respectively, so that partition noise is not inhibited. The electron and hole incoming currents, $I_{0}^{(e)}=\mathcal{P}_{1} h \nu e / h$ and $I_{0}^{(h)}=-I_{0}^{(e)}$, are sources of independent current fluctuations $\overline{\Delta I^{(e)^{2}}}=2 e I_{0}^{(e)} D(1-D) \Delta f$ and $\overline{\Delta I^{(h)^{2}}}=\overline{\Delta I^{(e)^{2}}}$ respectively. They add incoherently to give the total shot noise: $\overline{\Delta I^{2}}=4 h \nu \frac{e^{2}}{h} D(1-D) \mathcal{P}_{1} \Delta f$. In this process the photon energy quantum $h \nu$ plays the role of the bias voltage.

This is the basic shot noise mechanism generalized to 
multiple modes and multiple photon absorption processes that we have investigated experimentally. A complete formula for photo-assisted shot noise, with and without voltage bias, has been derived in 17,18 . For finite voltage, the formula predicts a singularity in the shot noise derivative at $e V=h \nu$ which was observed by the Yale group [7, 14] in a diffusive sample, definitely showing the existence of photo-assisted processes. The technique however measured the derivative of the noise with bias and could not measure the full shot noise in the zero current regime. Also it was not possible to vary the transmission for an accurate test of the theory. Here, we report total noise measurements. We show that electrons pumped to higher energy by photo-absorption do generate shot noise when no bias voltage is applied between reservoirs (and hence no current flows through the conductor). The sample, a Quantum Point Contact (QPC), allows one to vary the transmission and fully determine the Fano factor of the noise. In the doubly nonequilibrium regime, where both rf and finite bias voltage are applied, we recover the $e V=h \nu$ noise singularity providing further evidence that photo-pumping is the basic underlying mechanism.

The QPC is realized using a $2 \mathrm{D}$ electron gas in GaAs/AlGaAs with $810^{5} \mathrm{~cm}^{2} / \mathrm{Vs}$ mobility and $4.810^{11} \mathrm{~cm}^{-2}$ density. Special etching of the mesa prior to evaporation of the QPC metallic gates provides significant depletion at the $\mathrm{QPC}$ with zero gate voltage. At low temperature, well defined conductance plateaus for gate voltages ranging from $-55 \mathrm{mV}$ to $30 \mathrm{mV}$ allow accurately tuning of the transmission probability of the first two modes. The noise measurements were performed using a cross correlation technique 19] in the 2.6 to $4.2 \mathrm{kHz}$ range. The current noise power $S_{I}$ is calculated from the voltage noise power $S_{V}$ measured across the sample : $S_{I}=G^{2} S_{V}$ where $G$ is the differential conductance recorded simultaneously. A $5.2 \times 10^{-28} A^{2} / H z$ background current noise results from the amplifier current noise and the room temperature $100 \mathrm{M} \Omega$ current source. The sensitivity of our experimental setup is checked within $2 \%$ both by measuring the quantum reduction of shot noise [3, [4] at transmission $1 / 2$ and also by measuring the thermal noise for temperature varying from $200 \mathrm{mK}$ to $600 \mathrm{mK}$. The base electronic temperature is $94 \pm 5 \mathrm{mK}$ for a $28 \mathrm{mK}$ refrigerator temperature. The difference arises from the low loss coaxial cable carrying the rf which brings a wide bandwidth high temperature black body radiation to the sample [19.

The first step of our experiment is to determine the frequencies giving the highest coupling between the radiofrequency and the sample. This is achieved by measuring a weak photocurrent which never exceed $0.2 n \mathrm{~A}$ in the explored rf power range (the equivalent open circuit voltage is always lower than $k_{B} T$ such that its effect on noise can be neglected in the experiments described below). From this study we found a coupling sharply peaked at two frequencies 17.32 and $8.73 \mathrm{GHz}$ which therefore will be used in the following.

It is interesting to compare the rf period with the transit time of electrons between reservoirs. The distance between ohmic contacts being $30 \mu \mathrm{m}$ and the elastic mean free path $9 \mu \mathrm{m}$ we estimate the transit time to be $0.4 \mathrm{~ns}$. This is shorter than the coherence time and much longer than the rf period such that application of photo-assisted model is legitimate.

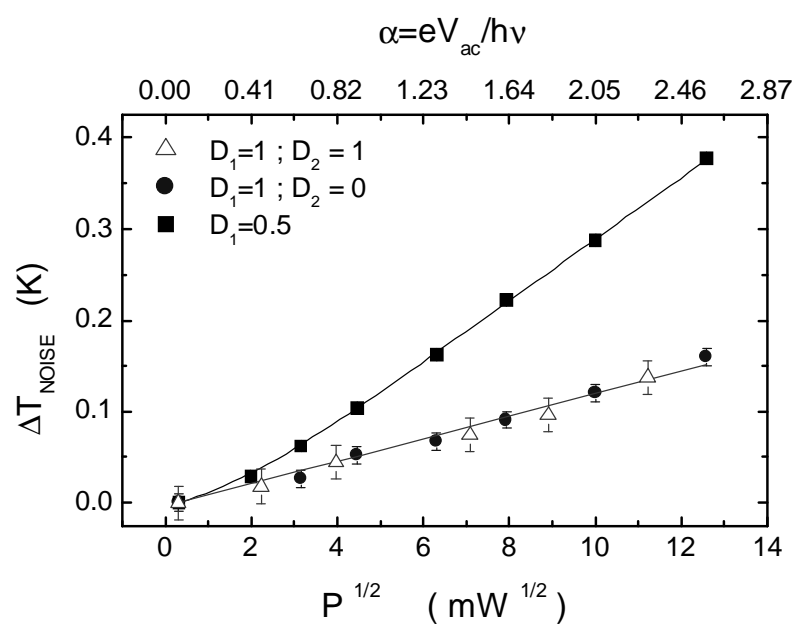

FIG. 1: Excess noise temperature as a function of the rf power $P$ on the top of the fridge at $17.32 \mathrm{GHz}$. From the noise increase at transmission 1 , we deduced the electronic temperature increase due to dissipation. The solid line for $D=0.5$ is a fit using Eq.(1) when taking into account the temperature increase. It gives the proportionality between $\alpha$ and $P^{1 / 2}$

We now present the results of our observation of photoassisted electron and hole partition noise with no applied bias voltage. In the limit where $h \nu \gg k_{B} T$, the noise formula 14, 17, 18 is :

$$
\begin{aligned}
T_{N} & =T\left(J_{0}^{2}(\alpha)+\frac{\sum_{n} D_{n}^{2}}{\sum_{n} D_{n}}\left(1-J_{0}^{2}(\alpha)\right)\right) \\
& +\sum_{l=1}^{+\infty} \frac{l h \nu}{k_{B}} J_{l}^{2}(\alpha) \frac{\sum_{n} D_{n}\left(1-D_{n}\right)}{\sum_{n} D_{n}}
\end{aligned}
$$

Here $D_{n}$ is the transmission probability of the $n^{\text {th }}$ mode, $\alpha=e V_{a c} / h \nu, J_{l}$ the integer Bessel function of order $l$ and $V_{a c}$ the rf voltage amplitude. The first term represent the thermal noise of unpumped and pumped electrons. The second term (which interests us here) is the partition noise of photo-created electrons and holes scattered by the QPC as discussed in the beginning $\left(\mathcal{P}_{1}=J_{1}^{2}\right.$ here). When the modes are either fully transmitted or reflected ( $D_{n}=1$ or 0 ), the noise is Johnson-Nyquist noise : $T_{N}=T$ and does not depend on rf power. However, in a real experiments heating of the reservoir by the $\mathrm{rf}$ power can not be excluded [14]. We thus first performed measurements on the first conductance plateau $\left(D_{1}=\right.$ 
$\left.G / G_{0}=1\right)$ and on the second plateau $\left(D_{1}=D_{2}=1\right.$, $G / G_{0}=2$, where $\left.G_{0}=2 e^{2} / h\right)$. An increase of the noise is indeed observed when increasing the rf power as shown in fig.(11). Here, and in the following, the current noise power is expressed in terms of noise temperature $T_{N}=$ $S_{I} / 4 G k_{B}$. Starting from a base electron temperature of $94 \mathrm{mK}$, we observe a small noise temperature increase which reaches $150 \mathrm{mK}$ for the highest power used in the experiments. The increase is the same on the first and second plateau. This indicates that heating occurs at the contact and is not related to the physics of scattering at the QPC. It is likely that heating of the contact results from rf absorption in the lossy coaxial lines.

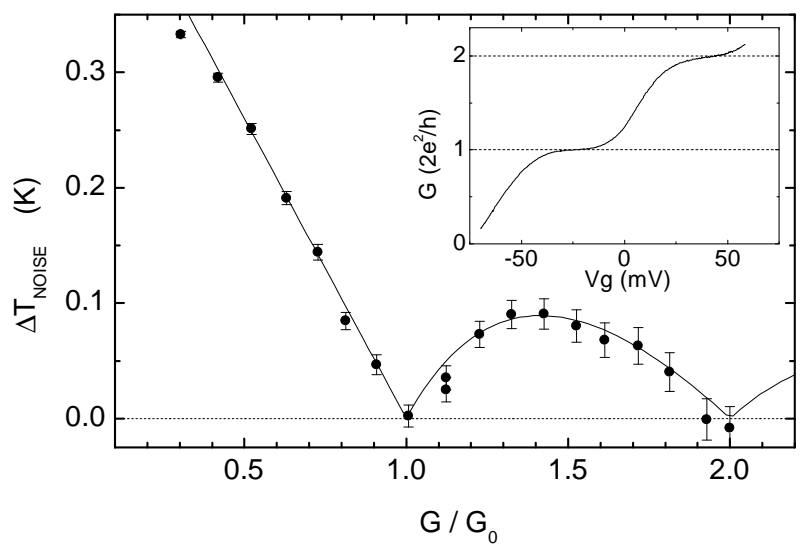

FIG. 2: Noise temperature increase as a function of the transmission $G / G_{0}$ when applying a $17.32 \mathrm{GHz}$ ac excitation with $\alpha=2.3$. The effect due to heating deduced from Fig.(11) has been removed. The solid line is the quantum suppression of the noise $\sum_{n} D_{n}\left(1-D_{n}\right) / \sum_{n} D_{n}$. Inset: conductance versus gate voltage.

Having characterized the heating, we focus on the partition noise regime expected for partial mode transmission. Fig.(1) shows a much larger increase of the noise temperature for $D_{1}=1 / 2$ than for $D_{1}=1$ and $D_{1}=D_{2}=1$. Can this difference to be attributed to partition noise of photo-pumped electrons or to thermally assisted shot noise? It is straightforward to give a quantitative estimation of the latter process. For an average temperature increase of the left and right reservoirs $\Delta T=\left(\Delta T_{\text {left }}+\Delta T_{\text {right }}\right) / 2$, one finds that the noise temperature increase never exceeds $\Delta T_{N}^{t h} \leq \Delta T+\left(1-D_{1}\right)[2 \ln 2-1] \Delta T$. According to the study at transmission 1 and $2, \Delta T$ is at most $150 \mathrm{mK}$, which gives $\Delta T_{N}^{t h} \leq 179 \mathrm{mK}$ at half transmission, i.e. only $29 \mathrm{mK}$ above the noise temperature increase observed on the plateaus. The much larger noise observed at $D_{1}=1 / 2$ strongly suggests the presence of photoassisted process. Taking the heating into account Eq.(11) fits the experimental results extremely well. From this we deduce $\alpha=e V_{a c} / h \nu$ to be a function of the square root of the applied rf power. This provides a calibration of the rf coupling which will be used below [20].
To fully characterize the partition noise of photopumped electrons and holes a systematic study as a function of transmission has been performed. The transmissions are measured using simultaneous measurement of the conductance $G=G_{0} \sum_{n} D_{n}$. Fig.(2) shows the noise temperature variation versus transmission for $\alpha=2.3$. The first term of Eq.(1) has been subtracted from the data as the transmissions $D_{n}, \alpha$ and the dependence of the electronic temperature with rf power are known. This allows better comparison with the second term of Eq.(1) which represents the electron hole partition noise. The variation of the noise is clearly proportional to the Fano factor [3, 国 $\sum_{n} D_{n}\left(1-D_{n}\right) / \sum_{n} D_{n}$ and unambiguously demonstrates the photo-assisted partition noise. Indeed the solid curve is the theoretical comparison with no adjustable parameter. Fig.(2) is the central result of this present work. This is the first observation of electron hole partition noise without net electron transport.
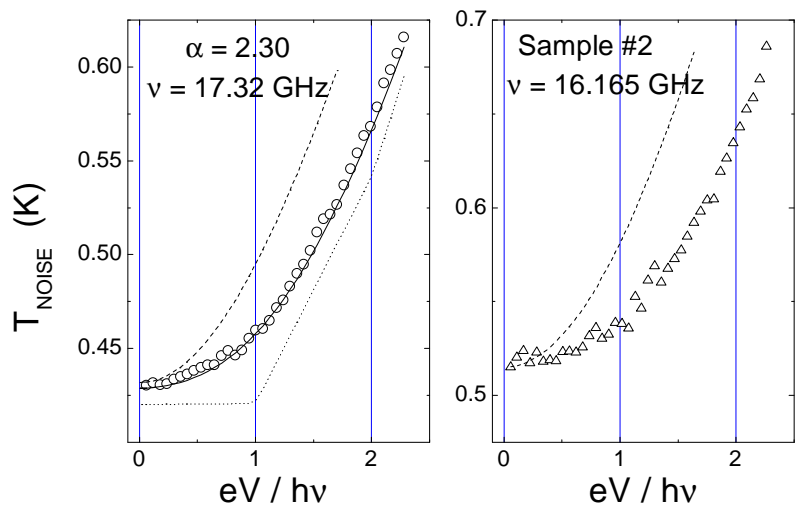

FIG. 3: Left graph: Noise temperature as a function of $e V / h \nu$ with $17.32 \mathrm{GHz}$ ac excitation. The measured electron temperature was $229 \mathrm{mK}$ and $\alpha=2.3$. The dotted line is the expected photo-assisted noise at zero temperature with $\alpha=2.3$ shifted for comparison. The dashed line is the non photoassisted shot noise with $T=430 \mathrm{mK}$. Continuous line is the photo-assisted noise calculated using Eq.(2) without adjustable parameter. Right graph: Noise temperature as a function of $e V / h \nu$ with $16.165 \mathrm{GHz}$ ac excitation for a different sample. The dashed line is the expected non photoassisted shot noise for $T=520 \mathrm{mK}$.

A further check that photo-assisted noise is the basic underlying mechanism is to apply both rf and finite bias voltage simultaneously on the QPC. It is known that a singularity occurs in the shot noise variation at $\mathrm{eV}=h \nu$ [7. 14, 17, 18]. This is simple to understand. Lets assume that the left reservoir has a chemical potential rise $\mathrm{eV}$ above the right. The noise arises from transport noise of pumped and unpumped electrons in the energy window $\mathrm{eV}$ and from photo-pumped electron hole pairs in the energy window $h \nu-e V$. The latter process observed previously at zero bias, disappears for $e V \geq h \nu$ leading to the singularity. The complete formula including multiple photon processes is [17: 


$$
T_{N}=T \frac{\sum_{n} D_{n}^{2}}{\sum_{n} D_{n}}+\frac{\sum_{n} D_{n}\left(1-D_{n}\right)}{\sum_{n} D_{n}} \sum_{ \pm} \sum_{l=0}^{+\infty} J_{l}^{2}(\alpha) \frac{e V \pm l h \nu}{2 k_{B}} \operatorname{coth}\left(\frac{e V \pm l h \nu}{2 k_{B} T}\right)
$$
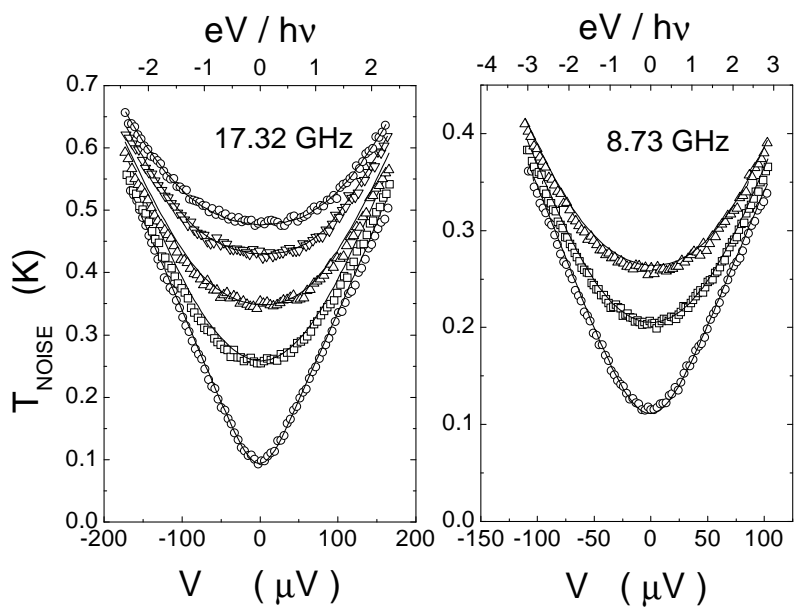

FIG. 4: Noise versus bias voltage. The solid lines are the theoretical curves with $\alpha$ and $T$ deduced from the equilibrium noise under rf illumination (see Fig.11). For the $17.32 \mathrm{GHz}$ curves, $\alpha=0.065 ; 1.29 ; 1.83 ; 2.30 ; 2.58$ and $T=$ $94 ; 168 ; 200 ; 229 ; 246 \mathrm{mK}$ and for the $8.73 \mathrm{GHz}$ curves, $\alpha=$ $0.51 ; 1.81 ; 2.56$ and $T=105.5 ; 145 ; 167.7 m K$.

Fig.(3), left graph, shows total noise measurements versus bias voltage at transmission $1 / 2$ for the same conditions as Fig.(2) $(\alpha=2.3, T=229 \mathrm{mK})$. As we can see on Fig.(3) for bias higher than $h \nu / e$ the noise starts to increase more rapidly, a hallmark of photo-assisted processes. This behavior cannot be attributed to simple thermal rounding, even if we assume that the $V=0$ noise temperature was corresponding to $430 \mathrm{mK}$ electronic temperature (dashed line). In order to better reveal the expected singularities in the voltage, we have plotted the noise at $T \approx 0$ (dotted curve). As all parameters $\alpha, T$, and $D$ are known we can use Eq.(2) to make a comparison with our data. The agreement is excellent. Even the thermal rounding of the singularity at $\mathrm{eV}=h \nu$ is well reproduced. The singularity of noise has been also observed using a different sample with a slightly different coupling and pumping frequency and is displayed on the right graph of Fig.(3).

Finally Fig.(14) shows a set of curves for various rf power values at 17.32 and $8.73 \mathrm{GHz}$ [21]. The curves show that for the two different frequencies, the voltage scale of noise variation is determined by the photon energy quantum $h \nu$ and not by the thermal energy scale. From the regime of nearly pure shot noise to the regime of strongly photo-assisted shot noise all curves compares accurately with theory without any adjustable parameter.
To summarize, absolute noise measurements on a quantum point contact under rf irradiation have provided the first demonstration that the quantum partition noise of electrons can be observed when no current flows through the sample. This is possible because photo created electron hole pairs scatters at the point contact generating current fluctuations. The photo-assisted process has been further brought into evidence when applying finite voltage leading to singularities for $\mathrm{eV}=h \nu$. All data show perfect agreement with the quantum scattering theory of photo-assisted shot noise.

* Also at Laboratoire de Physique de la Matière Condensée, Ecole Normale Supérieure, Paris.

[1] M. J. M. de Jong and C. W. J. Beenakker, edited by L. L. Sohn, L. P. Kouwenhoven, and G. Schön (Kluwer, Dordrecht, 1997).

[2] Y. M. Blanter and M. Büttiker, Phys. Rep. 336, (2000).

[3] M. Reznikov et al., Phys. Rev. Lett. 75, 3340 (1995).

[4] A. Kumar et al., Phys. Rev. Lett. 76, 2778 (1996).

[5] L. Saminadayar et al., Phys. Rev. Lett. 79, 2526 (1997).

[6] R. de Picciotto et al., Nature 389, (1997).

[7] A. A. Kozhevnikov, R. J. Schoelkopf, and D. E. Prober, Phys. Rev. Lett. 84, 3398 (2000).

[8] X. Jehl et al., Nature 405, 50 (2000).

[9] P. Dieleman et al., Phys. Rev. Lett. 79, 3486 (1997).

[10] R. Cron et al., Phys. Rev. Lett. 86, 4104 (2001).

[11] A. H. Steinbach, J. M. Martinis, and M. H. Devoret, Phys. Rev. Lett. 76, 3806 (1996).

[12] M. Henny et al., Phys. Rev. B 56, 2871 (1999).

[13] S. Oberholzer, E. V. Sukhorukov, and C. Schönenberger, Nature 415, 567 (2002).

[14] R. J. Schoelkopf et al., Phys. Rev. Lett. 80, 2437 (1998).

[15] G. B. Lesovik, Pisma Zh. Eksp. Teor. Fiz. 49, 513 (1989) [Sov. Phys. JETP Lett. 49, 592 (1989)]; M. Büttiker, Phys. Rev. Lett. 65, 2901 (1990); Th. Martin and R. Landauer, Phys. Rev. B 45, 1742 (1992).

[16] E. V. Sukhorukov and D. Loss, Phys. Rev. B 59, 13054 (1999).

[17] G. B. Lesovik and L. S. Levitov, Phys. Rev. Lett. 72, 538 (1994).

[18] M. H. Pedersen and M. Buttiker, Phys. Rev. B 58, 12993 (1998).

[19] D. C. Glattli et al., J. Appl. Phys. 81, 7350 (1997).

[20] Referred to the power $P$ delivered by the rf source before attenuation, we find a proportionality between $\alpha$ and $10^{P(d B m) / 20}$ equal to $0.204 \pm 0.004$ at $17.32 \mathrm{GHz}$ and $1.5 \pm 0.1$ at $8.73 \mathrm{GHz}$.

[21] The weak photocurrent $I_{p h}$ gives an offset in $V$ which is less than $6 \mu \mathrm{V}$ for $D=0.5$. This offset is removed. 